\title{
Frontières
}

\section{Quels enjeux contemporains autour du cadavre et des cendres?}

\section{Gaëlle Clavandier}

Volume 23, numéro 1, automne 2010

Enquêtes sur le cadavre : 1 . Fascination

URI : https://id.erudit.org/iderudit/1004018ar

DOI : https://doi.org/10.7202/1004018ar

Aller au sommaire du numéro

\section{Éditeur(s)}

Université du Québec à Montréal

ISSN

1916-0976 (numérique)

Découvrir la revue

Citer cet article

Clavandier, G. (2010). Quels enjeux contemporains autour du cadavre et des cendres ? Frontières, 23(1), 14-18. https://doi.org/10.7202/1004018ar
Résumé de l'article

Les nouvelles prises en charge du cadavre ont beaucoup à nous dire de notre rapport contemporain à la mort. S'il est acquis que la ritualisation traditionnelle, figure organisatrice des funérailles, est désormais remplacée par d'autres formes de socialisation, reste néanmoins à définir quel rôle on entend faire jouer à ce corps qui n'est plus en vie. Traditionnellement, au travers du cadavre et des mutations qu'il subit, c'est la rupture qui était signifiée grâce à l'élaboration d'un rapport à la mort. Étant médié, ce rapport créait de la distance, non de la proximité. Aujourd'hui, en plaçant le corps mort comme entité à part entière non disjointe de la vie, c'est la place faite à la mort qui est en question. Qu'en est-il alors de l'étape incontournable de la " séparation " ? À partir d'une réflexion sur le statut et la destination des cendres, à laquelle nous adjoindrons d'autres illustrations, nous montrerons que toutes participent d'une entreprise de faire corps avec le mort. Conduite au nom de la sécurisation des pratiques et du respect dû au corps, cette nouvelle façon d'envisager les « restes » indique que la frontière autrefois intangible entre la vie et la mort est en train d'exploser.
Ce document est protégé par la loi sur le droit d'auteur. L'utilisation des services d’Érudit (y compris la reproduction) est assujettie à sa politique d'utilisation que vous pouvez consulter en ligne.

https://apropos.erudit.org/fr/usagers/politique-dutilisation/ 
Résumé

Les nouvelles prises en charge du cadavre ont beaucoup à nous dire de notre rapport contemporain à la mort. S'il est acquis que la ritualisation traditionnelle, figure organisatrice des funérailles, est désormais remplacée par d'autres formes de socialisation, reste néanmoins à définir quel rôle on entend faire jouer à ce corps qui n'est plus en vie. Traditionnellement, au travers du cadavre et des mutations qu'il subit, c'est la rupture qui était signifiée grâce à l'élaboration d'un rapport à la mort. Étant médié, ce rapport créait de la distance, non de la proximité. Aujourd'hui, en plaçant le corps mort comme entité à part entière non disjointe de la vie, c'est la place faite à la mort qui est en question. Qu'en est-il alors de l'étape incontournable de la «séparation » ? À partir d'une réflexion sur le statut et la destination des cendres, à laquelle nous adjoindrons d'autres illustrations, nous montrerons que toutes participent d'une entreprise de faire corps avec le mort. Conduite au nom de la sécurisation des pratiques et du respect dû au corps, cette nouvelle façon d'envisager les "restes» indique que la frontière autrefois intangible entre la vie et la mort est en train d'exploser.

Mots clés: cadavre - corps - cendres rituels - législation funéraire.

\section{Abstract}

The new ways in which we deal with corpses have a lot to say about our current relationship to death. If we accept that traditional ritualization, which is what drives the organisation of funerals, has now been replaced by other forms of socialization, we still need to define, nonetheless, the role we intend the dead body to play. Traditionally, through the corpse itself and the transformations it had to undergo, it was the break, the severing of a relationship, which was signified thanks to the elaboration of a relationship to death. Having been mediated, this relationship created distance, not closeness. Today, by placing completely to one side the dead body as an entity not disconnected from life, it is the place reserved for death which is in question. What happens now with the unavoidable stage of "separation" ? Beginning with observations on the status and the destination of human ashes, to which I will add other illustrations, I will show that they all stem from an attempt to form one body with the dead. Operating under the name of observance and respect for the body, this new manner of contemplating the "remains" indicates that the once intangible line between life and death is currently being obliterated.

Keywords: corpse - body - ashes rituals - funerary legislation.

\section{Quels enjeux contemporains autour du cadavre et des cendres?}

\section{Gaëlle Clavandier, \\ maître de conférences, Université Jean-Monnet de Saint-Étienne, chercheure au Centre Max-Weber, CNRS, Université de Lyon.}

Qui mieux que le cadavre traduit le sentiment d'horreur provoqué par la mort? Référent lourd de sens puisqu'il énonce que le corps en vie n'est plus, sa présence est néanmoins nécessaire afin que le processus de deuil puisse débuter (Thomas, 1980). Si cette constante est identifiable pour toutes les cultures, reste que la période contemporaine, au travers des changements qu'elle porte, contraint à renouveler les questionnements. Parmi les transformations notables, on observe une ambivalence entre la sur-présence des cadavres, lesquels stimulent notre imaginaire dans les séries télévisées, les romans policiers ou encore les faits divers et la disparition quasi parfaite du cadavre dans les pratiques funéraires, puisque avec les soins de conservation notamment, c'est l'image d'un corps en vie qui est restituée à la famille éprouvée (Baudry, 1999, p. 123-142).

C'est dans ce contexte générique que nous nous attacherons à analyser la relation qu'entretiennent cadavres et cendres dans les pratiques funéraires contemporaines, et ce, tant à l'échelle du cadre réglementaire et légal qu'à celle des ajustements des professionnels et des familles. Très ancrée en France, la «culture de la trace» (Urbain, 1989, p. 289-336) s'élabore à partir d'un cadavre que l'on inhume dans un cimetière public, étape indispensable à la «séparation » (Thomas, 1985, p. 169219). Or, cette culture est aujourd'hui mise en cause par la montée en puissance de la pratique crématiste, sans que l'on sache pour l'heure si les transformations des usages induiront des variations infimes ou au contraire une mutation durable. Comme l'inhumation, elle pose la question du devenir du corps et de son statut post mortem, mais au contraire de la première, elle offre plusieurs alternatives en matière de «destination» du substrat corporel, ici les cendres. Ces options sont rendues possibles par une «manipulation» plus aisée des cendres qui, paradoxalement, restent mobiles en raison de leur état, mais stables par leur imputrescibilité (à la différence du cadavre qui, lui, subit une lente mutation). On le verra, cet écheveau de pratiques, si diversifié soit-il, n'en constitue pas moins 
CHACUNE À LEUR ÉCHELLE, LA TOILETTE MORTUAIRE, LA CRÉMATION, LA MOMIFICATION, L'INHUMATION... AVAIENT POUR FONCTION PREMIÈRE DE CRÉER UNE LIMITE DURABLE ENTRE LA BIOLOGIE D'UNE PART ET L'ANTHROPOLOGIE D'AUTRE PART, EN FAISANT DU CADAVRE LA PIERRE ANGULAIRE DES RITUELS FUNÉRAIRES D'ADIEU.

un assemblage qui tient grâce à une lente élaboration culturelle. Par réfraction, les interrogations sur le cadavre sont centrales puisqu'elles supposent de questionner tant le rapport à la mort que le statut du corps lui-même, nécessitant de dépasser le cadre des pratiques funéraires. Objet central de notre rapport au monde, bien qu'il soit le plus souvent masqué, le cadavre oblige à s'interroger sur les limites - au sens plein du terme - de l'humain (contours physiologiques, frontières spatiales, cadres réglementaires, repères culturels et cultuels, seuils émotionnels et intellectuels).

CADAVRES: DE L'OBJET DE FICTION À L'IMAGE DU CORPS MORT

Quand bien même l'objet questionné ici est relativement précis ${ }^{1}$, il nous paraît essentiel de nous concentrer sur l'apparente contradiction entre une recrudescence de la présence des cadavres sur la scène médiatique et leur retrait de la scène mortuaire à proprement parler. Cette incise permettra de replacer les enjeux d'une réflexion sur le cadavre et surtout de revenir sur les ambiguiités maintes fois soulevées par nos prédécesseurs.

D'un côté, il y aurait une surenchère bien orchestrée au sujet des cadavres, qui de simples figurants jusque dans les années 1990, seraient passés au rang des personnages principaux. Le cadavre aurait désormais un message à nous livrer, à l'instar de ces ossements qui permettent de mener l'enquête, de mieux connaître la victime et son environnement et de remonter immanquablement jusqu'à la scène du crime et presque accessoirement au criminel. De l'anthropologie médicolégale (Koff, 2004) à l'univers fictionnel des séries télévisées, il n'y a qu'un pas. Très visible dans les séries américaines des années 2000, la fiction accorde désormais une place importante à la victime et relègue le criminel à une position secondaire, si bien que la narration se nourrit de l'enquête. Ces séries modernes révolutionnent le genre policier et favorisent l'émergence d'un sous-genre que P. Mercklé et T. Dollé nomment les «séries macabres». «Encyclopédie illustrée des façons de mourir », ces séries restent néanmoins des fictions et s'élaborent à partir d'une gram- maire spécifique (Mercklé et Dollé, 2009). Certes, le corps-cadavre irradie par sa présence, mais celle-ci obéit à un ensemble de règles qui définissent les limites de ce qui peut être vu: les parties génitales sont par exemple protégées du regard. À un autre niveau, tout ce qui touche au corps de l'enfant doit revêtir une forme de sacralisation. Ainsi, le parcours du cadavre, de la scène du crime, où il est un corps impur, à la morgue, où il devient corps mort par la blancheur du lieu qui le lave de l'infamie, est balisé par une série d'étapes qui viennent signifier une réappropriation du corps. Cette reconquête passe par les mains d'autres «corps » cette fois institutionnels, médecins, enquêteurs, famille et proches...

D'un autre côté, et dans le même temps, le cadavre est réduit au silence, à la nécessité de s'oublier derrière la façade que constitue le corps, unique résidu acceptable lorsque la mort survient. Propriété incontestable de la Société de conservation, seul le corps a droit de cité lorsque la mort se présente et reste le principal support du souvenir (Urbain, 1978). Quand le cadavre renvoie au processus de thanatomorphose et par effet à l'impur, le corps mort, lui, paraît immuable et n'a de cesse d'être sacralisé. Ainsi, les pratiques funéraires effacent ce référent «biologique primitif» que constitue le cadavre pour en faire un être stabilisé, seul socialement et culturellement acceptable (Thomas, 1980). Chacune à leur échelle, la toilette mortuaire, la crémation, la momification, l'inhumation... avaient pour fonction première de créer une limite durable entre la biologie d'une part et l'anthropologie d'autre part, en faisant du cadavre la pierre angulaire des rituels funéraires d'adieu. Avec l'apparition de la thanatopraxie, la disparition du cadavre est consommée, puisque ce n'est plus à une mise en présence qu'est conviée la famille, mais à une ultime rencontre avec l'image parfaite du vivant $^{2}$. D'où la thèse de la disparition de la mort et par extension du cadavre, défendue par P. Baudry (2004), avec une mise en exergue de la vie.

Le contraste entre une valorisation $d u$ cadavre fictionnel et une dissimulation du cadavre «réel », si l'on peut dire, n'est donc pas aussi manifeste qu'il n'y paraît. Arrêtons-nous quelques instants sur cette apparente contradiction entre l'image d'un cadavre dont la présence itérative dans les séries télévisées en fait un objet hyperréaliste (un objet encyclopédique) et un cadavre qui tend à s'effacer peu à peu des pratiques funéraires, en laissant place à l'évanescence des cendres et à la figure iconique d'un corps en vie, devenant image du réel puisque masquant la dégradation future du corps. Ainsi, dans les deux cas, l'on assiste à un jeu de cacher-montrer ou, dit autrement, de mise en évidence et de dissimulation, jeu qui fait état qu'au sujet du cadavre, "imaginaire et réel» sont en constante redéfinition et que l'objet de la culture est d'en définir les frontières. Par essence objet culturel, le cadavre n'en a pas fini d'être configuré, montrant que sa «nature primitive» n'est pas aujourd'hui, plus qu'hier accessible à nos consciences, malgré le recours à la technique.

Perceptible pour les soins de conservation, mais aussi support des scénarios de fictions, la réappropriation du cadavre est également explicite à propos des cendres. Par réappropriation, nous entendons la capacité qu'ont (à des échelles différentes), une personne, une famille, une communauté ou un groupe social, une société à l'échelle d'un État, de définir, délimiter, maîtriser ou plutôt d'apprivoiser une réalité en soi irréductible comme le cadavre. De l'ordre de la traduction (puisqu'elle permet de «conduire au-delà »), cette opération repose tant sur une démarche expérimentale que critique. Elle implique pour le chercheur de tenir l'ensemble des entrées aussi bien celles qui touchent à l'élaboration d'un cadre juridique et réglementaire que celles reliées à son appropriation par les professionnels du funéraire et des familles, sachant que les pratiques elles-mêmes induisent un renouveau des questionnements éthiques, légaux et politiques et, au final, une évolution des représentations.

Concrètement, la réflexion inhérente à la montée en puissance de la crémation en France, depuis les balbutiements des années 1980 jusqu'à l'extension actuelle de la pratique qui tend à la normaliser, suppose de s'interroger sur les conséquences factuelles de ces transformations (quelle destination pour les cendres?), tout autant que sur la «philosophie» qui les anime (quel statut accorder aux cendres et plus largement au corps mort?).

\section{CORPS, CADAVRES, CENDRES}

"L'homme perdure-t-il dans la chair, même lorsque celle-ci est désertée?» Question insondable en soi, la variété et la relativité des réponses est telle que per- 
sonne jusqu'à ce jour n'a pu se prononcer de façon définitive. Avec raison, D. Le Breton rappelle que, d'un point de vue anthropologique, le statut attribué au cadavre dépend du statut culturel de la mort (Le Breton, 2006). Ainsi, si nombre de cultures vont dissocier, dans une perspective «dualiste », corps et âme (ou esprit), il est plus délicat de tirer un trait entre corps et cadavre. Pourtant aucune société ne va assimiler le cadavre à une chose inerte, aucune n'y est indifférente (Thomas, 1980). Le cadavre maintient donc un lien étroit avec le corps. Il en conserve durant un temps les contours et renvoie irrémédiablement à la vie (à la personne de son vivant). Inversement le corps est le siège d'une mort annoncée, d'où la métaphore $\mathrm{du}$ «corps tombeau» (Detrez, 2002, p. 30).

Nous plaçant sur le plan de la société contemporaine et en particulier de la situation française, cette tension perceptible au niveau du cadavre, qui en fait un objet ambigu et résistant, l'est-elle à propos des cendres? Plus précisément, faisant l'économie de la putréfaction, cette tension estelle réduite en raison de la transformation rapide du corps? Revenons en arrière, du temps des premières sociétés crématistes; on s'apercevra alors que les préoccupations matérielles ont toujours accompagné les principes philosophiques de la pratique crématiste. L'idée est de préserver de l'horreur de la putréfaction, de rendre la terre aux vivants et de libérer les villes des miasmes des cimetières (Nonnis Vigilante, 2007). La cendre en "réduisant le corps» permettrait de se centrer exclusivement sur les vivants. Le cadavre est évacué, avec lui le cimetière. Au-delà des valeurs hygiénistes et des projets urbanistiques que cette démarche sous-tend, c'est la place traditionnellement faite au corps mort qui vacille. La crémation moderne a-t-elle pour dessein la «disparition physique totale du mort» (Urbain, 2005, p. 305), venant ainsi renforcer la tentation d'occulter, voire d'anéantir toutes les contraintes inhérentes à la mort perceptible dans les idéaux des Libres penseurs? Les faits semblent pour certains entériner cette dématérialisation, avec par exemple le recours massif à la crémation pour les personnes atteintes du sida. D'elles-mêmes, elles ont intégré cette requête sans qu'elle ne soit pour autant réglementaire, comme pour signifier que le corps meurtri et stigmatisé est assimilable à un déchet. Cependant, il faut être prudent, d'une part, parce que la crémation peut s'accompagner de rituels profanes et avoir pour vocation une visée purificatrice (Broqua et al., 1998; Urbain, 1998; Fellous, 1998) et, d'autre part, car l'imaginaire de la dispersion peut aussi être empli de sens. En interrogeant les per- sonnes qui ont eu recours à un testament crématiste, V. Souffron montre que pour la plupart d'entre elles, il est «question de restituer l'être au monde» sous la forme d'un anonymat total ou celle d'une communion avec l'ensemble des morts et par extension avec le monde (Souffron, 2004, p. 298).

Comme pour le cadavre, le flottement qui tend à assimiler le corps mort à un corps est palpable, tout en étant exacerbé. Si le cadavre est un reste humain et à ce titre a des droits, même s'il peut être autopsié, disséqué, morcelé, les cendres quant à elles n'étaient pas, jusqu'à il y a peu, reconnues comme des «restes » à part entière. Pour toutes ces raisons, les usages en étaient démultipliés. De la dispersion en pleine nature à la conservation sur le buffet

\section{LE DÉBAT S'EST ÉGALEMENT}

PORTÉ SUR LES COMPORTEMENTS

\section{À L'ÉGARD DES CENDRES}

ET EN PARTICULIER SUR

LEUR DESTINATION. DOIT-ON LES

CONSERVER OU AU CONTRAIRE

\section{LES DISPERSER ? PEUT-ON}

EN FAIRE UN USAGE PRIVÉ ?

familial, en passant par un dépôt au cimetière ou la dispersion dans le «jardin du souvenir » toutes les configurations étaient envisageables. Des plus «raisonnables» aux plus fantasques: division ou amalgame des cendres, transformation en matériaux (diamant artificiel) ou en œuvre d'art, abandon dans une décharge, aux abords d'une route..., l'ensemble de ces pratiques était très éloigné de l'injonction faite au cadavre de demeurer dans le cimetière.

Sans être extravagants, ces différents usages - et la «permissivité» du droit à leur égard - montrent, s'il y a lieu, que les préconisations relatives aux cendres sont d'une tout autre nature que celles imposées au cadavre. Dès lors, la dangerosité que l'on attribue à ce dernier n'est pas par mimétisme toute transférée dans les cendres, lesquelles peuvent être disséminées et contaminer l'espace des vivants. Sous forme humoristique, Hardy et Cauvin, créateurs du personnage Pierre Tombal, montrent ce que pourrait être un monde où les ossements sont dispersés au même titre que le sont les cendres. Le résultat est saisissant. Voir les restes ainsi répan- dus laisse perplexe et suppose l'intervention d'un agent des forces de l'ordre pour mettre un terme à cette contamination de l'espace, alors que l'indifférence règne à l'égard des cendres. Tandis que les cendres se dispersent, se volatilisent et ne font sens en l'absence de support symbolique ${ }^{3}$, l'ossement, lui, demeure en raison de sa matérialité. Par extension, sa partie vaut pour le tout et le moindre os évoque le squelette et par prolongement le corps. Par effet, le cadavre est singulier, les cendres plurielles.

Cette «contradiction» entre une prise en charge très cadrée pour le cadavre et la souplesse d'intervention lorsque les cendres sortent du crématorium a soulevé nombre de questions sur la nature des rituels ou plus largement des usages funéraires à inventer et sur le statut à accorder aux cendres. Quelles que soient les modalités de ce questionnement - problématiques scientifiques, débats philosophiques, juridiques et éthiques, normes professionnelles, discussions informelles, œuvres littéraires ou plastiques -, toutes se saisissent d'un (ou plusieurs) problèmes et tentent de l'expliciter. À son échelle, le législateur entreprend de donner un cadre qui édifie le socle des pratiques tout en les «sécurisant» et repense le lien entre corps, cadavre et cendres.

\section{LE « RESPECT » DÛ AU CORPS}

Depuis les années 2000, notant des actes déviants, ou pour le moins une grande diversité des usages, différentes commissions parlementaires ont siégé pour échanger sur ce sujet (Nonnis Vigilante, 2007 ; Clavandier, 2008). Précisément, il a été question de statuer sur les cendres, ce qui a rendu nécessaire une réflexion sur le statut du cadavre. Le débat s'est également porté sur les comportements à l'égard des cendres et en particulier sur leur destination. Doit-on les conserver ou au contraire les disperser? Peut-on en faire un usage privé? Est-il nécessaire de créer de nouveaux rituels funéraires pour accompagner cet ensemble de pratiques qui éprouve la culture traditionnelle de la «trace» et de «l'enracinement» des corps?

Il y a quelques mois encore, la législation française était l'une des plus souples à ce sujet (Py et Mayer, 2008). Depuis la loi du 19 décembre 2008 relative à la législation funéraire, de nombreuses recommandations ont été apportées, lesquelles viennent spécifier le cadre légal de la pratique crématiste. Certaines touchent à son organisation: obligation d'édifier un site cinéraire dans les cimetières des communes (ou établissements publics de coopération intercommunale) de plus de 10000 habitants ; instauration de schémas 
régionaux afin d'harmoniser la politique de création des crématoriums. D'autres sont relatives à la destination des cendres, dont le dépôt privatif est désormais proscrit, et à leur statut. Avant toute chose, nous tenons à préciser que, plus que la loi elle-même, c'est l'esprit dans laquelle elle a été élaborée qui nous intéresse ici. Nous le verrons, cette précision est importante dans la mesure où elle permet de comprendre pourquoi le législateur a travaillé par homologie, comme si la proximité entre inhumation et crémation était un fait. Comme si la crémation, au travers des valeurs qu'elle infère comme des pratiques qu'elle occasionne, devait être (re)définie à partir de l'essaimage d'une culture déjà édifiée, à savoir la culture du souvenir des morts, qui prend sens dans un cadre laïc et républicain ${ }^{4}$.

Or, contre toute attente, le simple fait de penser la pratique crématiste a impliqué de redéfinir les pratiques funéraires dans leur ensemble. Ainsi, contrairement à une idée répandue qui relève d'une sécularisation des préceptes chrétiens par le droit, le cadavre n'avait pas de réel statut dans le droit français (Belhassen, 1997). Désormais, c'est chose faite, mais il aura fallu que les cendres deviennent un problème public pour que la loi soit modifiée en ce sens. Les articles 16 et $16-1$ du code civil français (à propos des droits civils des personnes et du respect du corps humain), qui définissent respectivement les droits de la personne ${ }^{5}$ et ceux de son $\operatorname{corps}^{6}$, ont été complétés par un article qui vient spécifier les droits relatifs aux «restes»: «Le respect dû au corps humain ne cesse pas avec la mort. Les restes des personnes décédées, y compris les cendres de celles dont le corps a donné lieu à crémation, doivent être traités avec respect, dignité et décence»(Art. 16-1-1). Dès lors, le corps, le cadavre et les cendres sont soumis au même ensemble de préconisations et ont les mêmes droits.

À un autre niveau, celui de la destination des cendres, la position du législateur a évolué touche par touche, mais a toujours été portée par deux principes qui émanent de la refonte du droit funéraire de la fin du $\mathrm{XIX}^{\mathrm{e}}$ et début $\mathrm{XX}^{\mathrm{e}}$ siècles. Certes non contradictoires, ces principes sont néanmoins ambivalents. Ils se fondent sur la liberté des funérailles (quant au mode de sépulture notamment) et sur le caractère public, laïc et républicain des obsèques, lequel a pour projet de susciter un souvenir en commun (le cimetière étant l'espace de ce souvenir). On comprend pourquoi la détention d'une urne à titre privatif pouvait se justifier en raison du principe de liberté (principe cher aux associations crématistes), mais était contraire au principe d'égalité d'accès au

recueillement. En faisant du cimetière le lieu «naturel » de destination des corps et des cendres (Clavandier, 2007), le législateur restreint l'éventail des usages, tout en assimilant de manière directe ou allusive cadavre et cendres, en les faisant devenir corps. Si cette assimilation est tangible au

\section{LE CORPS MORT DEVIENT UN PROCHE QU'IL FAUT}

SANS CESSE RECADRER, ALORS MÊME QUE TOUT L'ENJEU EST DE S'EN DÉTACHER. L'ÉPREUVE DE LA SÉPARATION, ÉTAPE INCONTOURNABLE DU DEUIL, EST AUJOURD'HUI MISE EN CAUSE.

niveau du législateur, elle l'est tout autant chez les opérateurs funéraires, qui font du cimetière, lieu de dépôt des "corps", le lieu de référence en matière funéraire, celui dont ils détiennent les clefs. Dans ce contexte, il n'est point surprenant de voir apparaître sur le marché des «monuments mixtes » visant à regrouper tous les corps (ceux inhumés et ceux crématisés) dans l'espace d'une même sépulture. À l'échelle des familles endeuillées, ce nouvel accommodement peut réduire la distance entre la réalisation des attentes personnelles, qui se formalisent par les "dernières volontés », et les habitudes et cérémonials familiaux qui peuvent être fermement ancrés dans une tradition. Il ne faut pas oublier qu'en matière funéraire les choix individuels (les vœux) sont mis en œuvre par un tiers, ce qui a un poids considérable. Sachant que ces vœux ont valeur testamentaire, celui qui doit les accomplir (aujourd'hui spécifié dans les textes par: "la personne ayant qualité pour pourvoir aux funérailles») peut entrer en conflit de valeurs avec les actes qu'il doit accomplir.

En ayant recours à la loi, le législateur avait à l'esprit de "sécuriser les pratiques » funéraires, un souhait qui vraisemblablement ne lui est pas propre, puisqu'il s'agit d'une demande récurrente des professionnels et des associations. À n'en point douter cet état d'esprit se répercutera sur l'ensemble des pratiques funéraires et aura, au final, un effet indéniable sur l'élaboration d'une culture funéraire plus normée.

\section{QUAND LA MORT FAIT « CORPS » AVEC LE VIVANT}

Ces dispositifs en émergence, avec au centre le cadavre, n'ont plus guère à voir avec les rites funéraires traditionnels. Ils ont pour objectif principal la «sécurisation » et la «protection» des individus, lequel se traduit par un encadrement des usages et l'édification d'un modèle de prise en charge. Ils se déplacent sur le champ de la morale et de l'éthique (le statut du scène de la dernière relation au vivant, c'était ensuite pour mieux s'en séparer (Thomas, 1985, p. 141-147). Désormais, le rapport au cadavre s'instruit sur un tout autre registre qui, en faisant de lui un corps, voire en faisant corps avec lui, a pour conséquence que la déliaison, pièce essentielle de la culture funéraire ancestrale, n'est plus envisageable.

En traçant le parcours des cendres (loi française du 19 décembre 2008), en reconnaissant le statut de personne à des fœtus nés sans vie sans prendre en compte le critère du développement (arrêts de la Cour de cassation du 6 février 2008), en interdisant l'exposition Our Body - $\bar{A}$ corps ouvert (référé du 21 avril 2009), chacune de ces réponses légales tendent à signifier le souci croissant de protéger le corps, en prenant en considération des dimensions humaines et éthiques, conformément à la demande des proches ou des militants associatifs. Sur des registres différents, en favorisant la reconnaissance sociale d'une demande plus intime, un nouveau rapport à la mort semble se dessiner (Déchaux, 2000 ; 2001), lequel a une incidence sur le corps lui-même. Cependant, il ne faut pas se tromper de cible en voyant dans cette transformation une exclusive "privatisation du mourir », la renvoyant à des aspects individuels. Par l'acte de nommer, dater, localiser, photographier, etc., la dimension collective n'est pas absente de ce processus, même si elle tend à changer de registre d'expression jusqu'à quitter presque entièrement la sphère rituelle et symbolique ${ }^{7}$.

Néanmoins, cet effet de présence que l'on constate au sujet du corps mort, avec tout l'environnement de préconisations, de valeurs et d'usages que cela représente, doit nous faire réfléchir au-delà même de cette évolution du cadre. Ainsi, l'on pourrait se satisfaire de repérer une évolution des formes de prise en charge du mourir, avec un basculement du rituel vers des formes de socialisation plus contemporaines. Pourtant, d'un point de vue anthro- 
pologique, cette «présence» pose question. D'une certaine manière, elle est contraire à toute entreprise de «neutralisation »de la mort, en voulant la faire sienne ${ }^{8}$. En intégrant la mort, dans le cycle vital ou dit autrement en faisant de la mort un avatar de la vie, la mise à distance, pièce mâ̂tresse des rituels funéraires, est réduite à néant (Baudry, 1999; 2004). De ce fait, en souhaitant faire du cadavre un corps (d'un point de vue factuel), voire en voulant faire corps avec lui (d'un point de vue éthique), il devient hasardeux d'établir une frontière claire entre vivants et morts et de permettre au mort de «mourir à sa vie ${ }^{9}$. Le corps mort devient un proche qu'il faut sans cesse recadrer, alors même que tout l'enjeu est de s'en détacher. L'épreuve de la séparation, étape incontournable du deuil, est aujourd'hui mise en cause. En raison même de cette incertitude latente, notre société (que ce soit à l'échelle des individus, mais également des professionnels) a du mal à lâcher prise au sujet des cadavrescorps, d'où leur sur-présence sur certaines scènes et leur absence sur d'autres ${ }^{10}$, d'où également cet aller-retour constant qui consiste à outiller les pratiques tout en permettant à la confusion de s'installer. Comme le disait très justement P. Baudry à propos de la thanatopraxie, d'un point de vue anthropologique, la mort est à la frontière de l'humain (Baudry, 1999). Penser que l'on peut composer avec elle de façon raisonnée et raisonnable est un non-sens, de même faire du cadavre un simple corps, certes mort, n'est pas sans risque.

S'il fallait conclure provisoirement sur la nouvelle place faite au corps mort, nous dirions qu'elle est paradoxale. Or, en tant que tel, le caractère ambivalent de cette posture pose problème puisqu'il répond par mimétisme à l'objet ambigu que constitue le cadavre, en introduisant du doute plutôt qu'une rupture. S'il a tendance à renforcer le cadre institutionnel (par l'intermédiaire des lois et des normes professionnelles notamment) et à insister sur le caractère impérieux d'une sécurisation des pratiques, il introduit dans le même temps la nécessaire reconnaissance des requêtes individuelles en accordant un crédit de plus en plus important à la personne, à son intégrité et à ses émotions. On imagine alors quelle interprétation peut être faite du cadre et comment celui-ci peut être flottant. Certes, apanage des sociétés démocratiques contemporaines, cette ouverture semble être gagnée sur les contraintes autrefois significatives, mais laisse place à une incertitude qui au sujet du cadavre n'est pas sans contrepartie.

\section{Bibliographie}

BAUDRY, P. (2004). Violences invisibles. Corps, monde urbain, singularité, Bègles, Éditions du Passant.

BAUDRY, P. (1999). La place des morts. Enjeux et rites, Paris, Armand Colin.

BELHASSEN, P. (1997). La crémation: le cadavre et la loi, Paris, LGDJ.

BROQUA, C., F. LOUX et P. PRADO (1998). "Sida: deuil, mémoire, nouveaux rituels", Ethnologie française, $\mathrm{n}^{\circ} 28$, p. 5-9.

CLAVANDIER, G. (2008). "Le droit contre les usages. La crémation, destination et statut des cendres », Quaderni, n 67, p. 107-124.

CLAVANDIER, G. (2007). «La crémation: des pratiques singulières à l'élaboration d'un cadre de référence», Études sur la mort, no 132 , p. 65-86.

DÉCHAUX, J.-H. (2001). "Un nouvel âge du mourir: "la mort en soi" ", Recherches sociologiques, $\mathrm{n}^{\circ} 32$, p. 79-100.

DÉCHAUX, J.-H. (2000). "L'“intimisation" de la mort. Note critique », Ethnologie française, vol. 30, p. 153-162.

DETREZ, C. (2002). La construction sociale du corps, Paris, Seuil, coll. «Points - Essai».

FELLOUS, M. (1998). «Le Quilt: un mémorial vivant pour les morts ", Ethnologie française, $\mathrm{n}^{\circ} 28$, p. 80-86.

KOFF, C. (2004). La mémoire des os, Paris, Éditions Héloïse d'Ormesson.

LE BRETON, D. (2006), "Le cadavre ambigu: approche anthropologique", Études sur la mort, no 126, p. 79-90.

MERCKLÉ, P. et T. DOLLÉ (2009). «Morts en séries: représentations et usages des cadavres dans la fiction télévisée contemporaine », Raison Publique, no 11, p. 226-246.

NONNIS VIGILANTE, S. (2007). «Encadrer la liberté: le statut et la destination des cendres dans le projet de réforme de la législation funéraire (2005-2006) », Quaderni, $\mathrm{n}^{\circ} 62$, p. 57-67.

PY, B. et M. MAYER (dir.) (2008). La crémation et le droit en Europe, Nancy, Presses Universitaires de Nancy.

SOUFFRON, V. (2004). «La crémation: de la pratique au rite? », dans S. PENNEC (dir.), Des vivants et des morts. Des constructions de la "bonne mort», Brest, ARS-CRBC, UBO, p. 293-301.

THOMAS, L.-V. (1985). Rites de mort. Pour la paix des vivants, Paris, Fayard.

THOMAS, L.-V. (1980). Le cadavre. De la biologie à l'anthropologie, Bruxelles, Éditions Complexe.

URBAIN, J.-D. (2005 [1989]). L'Archipel des morts. Cimetières et mémoire en Occident, Paris, Payot et Rivages.

URBAIN, J.-D. (1998). « Mort traquée, mort tracée. Culte des morts, crémation, sida», Ethnologie française, $\mathrm{n}^{\circ} 28$, p. 43-49.
URBAIN, J.-D. (1978). La Société de conservation. Sémiologie des cimetières en Occident, Paris, Payot.

\section{Notes}

1. L'objet est circonscrit aux pratiques funéraires et plus spécifiquement aux cendres, mais s'inscrit dans une problématique plus large sur le rapport entre corps et cadavre. Un rapport qu'il faut réinvestir en fonction des questionnements spécifiques que soulèvent les cendres.

2. Ici, le retour sur le vivant se fait, non pas sur l'origine ou même la description de la vie (le bios), mais sur l'image que l'on se fait du vivant.

3. Les cendres humaines n'ont pas d'attributs particuliers - à moins d'être spécialiste de la question - qui permettraient de les distinguer des cendres animales, végétales, ou même des déchets incinérés.

4. Dans cette perspective, malgré ses spécificités, la culture crématiste doit s'élaborer à partir des fondements d'une culture funéraire déjà identifiée qui a pour modèle l'inhumation, le cimetière, l'égalité entre les morts et le caractère public du souvenir.

5. «La loi assure la primauté de la personne, interdit toute atteinte à la dignité de celle-ci et garantit le respect de l'être humain dès le commencement de sa vie» (Art. 16 du code civil français).

6. «Chacun a droit au respect de son corps. Le corps humain est inviolable. Le corps humain, ses éléments et ses produits ne peuvent faire l'objet d'un droit patrimonial » (Art. 16-1 du code civil français).

7. L'exemple des fœtus nés sans vie est tout à fait significatif de ce travail de "socialisation». Avec la photographie post mortem, le choix du prénom, l'inscription à l'état civil (avec mention de l'heure et de la date de naissance), la mention sur le livret de famille, la reconnaissance de ses parents, l'accès à certains droits sociaux et la prise en charge du corps par sa famille en vue de ses obsèques, tous ces gestes et actes visent à faire du « foetus né sans vie » un enfant.

8. Intégrer la mort suppose de la faire sienne, ce qui revient à annihiler son altérité radicale, fondement ontologique et anthropologique du rapport à la mort. Dès lors, il ne s'agit plus d'une neutralisation, mais d'une tentative d'assimilation de la mort et plus précisément d'incorporation du cadavre.

9. D'où les expressions relevées par les anthropologues: «tuer le mort» (Baudry, 1999, p. 126), se libérer de «la mort-du-mort» (Balandier, 1988, p. 33).

10. Si l'on remarque une présence du cadavre dans les médias (informations et fictions), force est de constater qu'il est désormais quasi absent des pratiques funéraires. D'une part, les soins de conservations lui donnent l'aspect du vivant, d'autre part, la veillée du corps tend à disparaître et lors de la mise en bière de nombreuses précautions vont être prises pour éloigner les regards les plus vulnérables (par exemple les enfants). 